\title{
A geometric model for Hochschild homology of Soergel bimodules
}

\author{
BEN WEBSTER \\ GEORDIE WILLIAMSON
}

\begin{abstract}
An important step in the calculation of the triply graded link homology of Khovanov and Rozansky is the determination of the Hochschild homology of Soergel bimodules for $\operatorname{SL}(n)$. We present a geometric model for this Hochschild homology for any simple group $G$, as $B$-equivariant intersection cohomology of $B \times B$-orbit closures in $G$. We show that, in type A, these orbit closures are equivariantly formal for the conjugation $B$-action. We use this fact to show that, in the case where the corresponding orbit closure is smooth, this Hochschild homology is an exterior algebra over a polynomial ring on generators whose degree is explicitly determined by the geometry of the orbit closure, and to describe its Hilbert series, proving a conjecture of Jacob Rasmussen.
\end{abstract}

17B10; 57T10

\section{Introduction}

In this paper, we consider the Hochschild homology of Soergel bimodules, and construct a geometric interpretation of it. This will allow us to explicitly compute the Hochschild homology of a special class of Soergel bimodules.

Soergel bimodules are bimodules over a polynomial ring, which appear naturally both in the study of perverse sheaves on flag varieties and of the semiring of projective functors on the BGG category $\mathcal{O}$. Recently interest in them has been rekindled by the appearance of connections with link homology as shown by Khovanov [11].

Khovanov's work showed that one aspect of Soergel bimodules which had not been carefully studied up to that date was, in fact, of great importance: their Hochschild homology. While the operation of taking Hochschild homology is hard to motivate from a representation theoretic perspective, we argue that it is, in fact, naturally geometric.

Throughout, we let

- $G$ be a connected reductive algebraic group over $\mathbb{C}$, with Lie algebra $\mathfrak{g}$, 
- $B$ be a Borel subgroup of $G$,

- $T \subset B$ be a Cartan subgroup of $G$, with Lie algebra $\mathfrak{t}$,

- $n=\operatorname{dim} T$ be the rank of $G$,

- $W=N_{G}(T) / T$ be the Weyl group of $G$.

For any $w \in W$, we let $G_{w}=\overline{B w B}$.

As the closure of a group orbit, $G_{w}$ is an irreducible closed subvariety of $G$ which is smooth if and only if the corresponding Schubert variety $\mathcal{B}_{w}=G_{w} / B$ is (thus $G_{w}$ is typically singular). The subgroup $B \times B$ acts on $G_{w}$ by left and right multiplication. Restricting this action to the diagonal gives the action of $B$ by conjugation. Of course, we also have left and right actions of $B$ but we will never consider these separately. Whenever we refer to a $B$-action on $G_{w}$, we will always mean the conjugation action.

Given a complex algebraic variety $X$, we write $H^{*}(X)$ and $I H^{*}(X)$ for its cohomology and intersection cohomology with complex coefficients. If $X$ is in addition a $G$-variety we let $H_{G}^{*}(X)$ and $I H_{G}^{*}(X)$ denote the $G$-equivariant cohomology and $G$-equivariant intersection cohomology of $X$ (again with coefficients in $\mathbb{C}$ ) which is a module over $H_{G}^{*}(\mathrm{pt})$. We normalize intersection cohomology by requiring that $H^{*}(X) \cong I H^{*}(X)$ and $H_{G}^{*}(X) \cong I H_{G}^{*}(X)$ if $X$ is smooth.

We observe that $T$ is a deformation retract of $B$, so we have $H_{B}^{*}(X) \cong H_{T}^{*}(X)$ and $I H_{B}^{*}(X) \cong I H_{T}^{*}(X)$ for all $B$-spaces $X$. We will freely switch between $B$ - and $T$-equivariant cohomology and intersection cohomology throughout this paper.

Now consider the graded ring $S=H_{T}^{*}(\mathrm{pt})=\mathbb{C}\left[\mathfrak{t}^{*}\right]$ (where the elements of $\mathfrak{t}^{*}$ have degree 2), which is endowed with a $W$-action. Given a simple reflection $s \in W$, denote by $R_{S}$ the $S$-bimodule $S \otimes S^{s} S$ where $S^{s}$ is the subring of invariants under the reflection $s$. If $M$ is a graded $S$-bimodule denote by $M[a]$ the shifted module $(M[a])^{i}=M^{i+a}$. (Later we will think of Soergel bimodules as differential graded modules over $S \otimes S$, which is why we use $[a]$ to denote grading shift.)

We now come to the definition of Soergel bimodules:

Definition 1 We call an $S$-bimodule $R$ a Soergel bimodule if each indecomposable direct summand of $R$ is a direct summand of a tensor product of the form $R_{\mathrm{i}}[a]=R_{S} \otimes S$ $R_{t} \otimes_{S} \cdots \otimes_{S} R_{u}[a]$ in the category of graded $S$-bimodules, where $\mathbf{i}=(s, t, \ldots, u)$ is a sequence of (not necessarily distinct) simple reflections, and $a$ is an arbitrary grading shift.

The motivation for studying Soergel bimodules comes from representation theory, geometry and connections between the two. In fact, one has the following theorem: 
Theorem 1.1 (Soergel) The indecomposable Soergel bimodules are parametrized (up to a shift in the grading) by the Weyl group [15, Satz 6.14].

The indecomposable Soergel bimodule corresponding to $w \in W$ may be obtained as $R_{w} \cong I H_{B \times B}^{*}\left(G_{w}\right)[14$, Lemma 5].

Implicit in the theorem is the fact that $I H_{B \times B}^{*}\left(G_{w}\right)$ is a module over $H_{B \times B}^{*}(\mathrm{pt})=S \otimes S$ and hence may be regarded as an $S$-bimodule. More generally, Soergel bimodules can be identified with the $B \times B$-equivariant hypercohomology $\mathbb{H}_{B \times B}^{*}(G, \mathcal{F})$, where $\mathcal{F}$ is a direct sum of shifts of $B \times B$-equivariant simple perverse sheaves on $G$.

Our central result is a geometric description of Hochschild homology $H_{*}(-)$ of these bimodules.

Theorem 1.2 Let $\mathcal{F}$ be a semisimple $B \times B$-equivariant perverse sheaf on $G$ and let $R$ be its $B \times B$-equivariant cohomology. Then

$$
H H_{*}(R) \cong \mathbb{H}_{B}^{*}(G, \mathcal{F}),
$$

where $B$ acts by conjugation (ie, by the diagonal inclusion $B \hookrightarrow B \times B$ ). In particular, we have

$$
H H_{*}\left(R_{w}\right) \cong I H_{B}^{*}\left(G_{w}\right)
$$

Unfortunately, $I H_{B}^{*}\left(G_{w}\right)$ has a single grading, whereas $H H_{*}\left(R_{w}\right)$ has two: one by decomposition into the components $H H_{i}$ ("the Hochschild grading"), and one coming from the grading on $R_{w}$ ("the polynomial grading"). This isomorphism takes the single grading on $I H_{B}^{*}\left(G_{w}\right)$ to the difference of the two gradings on $H H_{*}\left(R_{w}\right)$.

We can give a geometric interpretation of these gradings, but in a somewhat roundabout manner, using the geometry of the intersection cohomology complex $\mathbf{I C}(B w B)$ (readers unfamiliar with intersection cohomology should refer to Section 3 and the references given there). We will first prove the following:

Theorem 1.3 Assume $G=\operatorname{SL}(n)$ or $\operatorname{GL}(n)$. Then the complex $\operatorname{IC}_{B}(B w B)$ is equivariantly formal. Thus, if we let $j_{B}: B=G_{e} \rightarrow G_{w}$ denote the inclusion, the map

$$
j_{B}^{*}: I H_{B}^{*}\left(G_{w}\right) \rightarrow \mathbb{H}_{B}^{*}\left(B, j_{B}^{*} \mathbf{I C}_{B}(B w B)\right)
$$

is injective and becomes an isomorphism after tensoring with the fraction field of $S$.

Applying our deformation retract from $B$ to $T$, we see that

$$
\mathbb{H}_{B}^{*}\left(B, j_{B}^{*} \mathbf{I C}_{B}(B w B)\right) \cong \mathbb{H}_{T}^{*}\left(T, j_{T}^{*} \mathbf{I C}_{B}(B w B)\right)
$$


where $T$ acts trivially on itself (that is, by the conjugation action). Thus by the Künneth theorem, we have a further isomorphism

$$
\mathbb{H}_{T}^{*}\left(T, j_{T}^{*} \mathbf{I C}_{T}(B w B)\right) \cong S \otimes_{\mathbb{C}} \mathbb{H}^{*}\left(T, j_{T}^{*} \mathbf{I C}(B w B)\right),
$$

which we can use to equip this $S$-module with a bigrading, with an "equivariant" grading from the first factor, and a "topological" grading from the second. We can use these to define "topological" and "equivariant" gradings on $H H_{*}\left(R_{w}\right)$, using transport of structure by the isomorphism of Theorem 1.2.

Thus, $H H_{*}\left(R_{w}\right)$ carries four natural gradings, which we call "Hochschild," "polynomial," "topological," and "equivariant."

Theorem 1.4 These gradings are related by the equations

$$
\operatorname{deg}_{t}(\gamma)=\operatorname{deg}_{h}(\gamma) \quad \operatorname{deg}_{e}(\gamma)=\operatorname{deg}_{p}(\gamma)-2 \operatorname{deg}_{h}(\gamma)
$$

where $\operatorname{deg}_{*}(x)$ denotes the degree of $x$ in the grading whose name begins with the letter $*$.

The case where $G_{w}$ is smooth is of special interest to us. Any reader who is unhappy with the presence of intersection cohomology and perverse sheaves may only consider the case where $G_{w}$ is smooth, in which case intersection cohomology is canonically isomorphic to Čech cohomology.

Theorem 1.5 If $G_{w}$ is smooth (in any type), then as a bigraded $S$-module, the Hochschild homology of $R_{w}$ is an exterior algebra over $S$. That is, we have an isomorphism

$$
H H_{*}\left(R_{w}\right)=\wedge^{\bullet}\left(\gamma_{1}, \ldots, \gamma_{n}\right) \otimes_{\mathbb{C}} S
$$

where $\left\{\gamma_{i}\right\}_{i=1, \ldots, m}$ are generators with

$$
\operatorname{deg}_{h}\left(\gamma_{i}\right)=1 \quad \operatorname{deg}_{p}\left(\gamma_{i}\right)=2 k_{i}
$$

for positive integers $k_{i}$ determined by the geometry of $G_{w}$.

These integers can be calculated using the action of $w$ on the root system or, in the case of $\operatorname{SL}(n)$, by presenting $G_{w} / B$ as an iterated Grassmannian bundle.

While the indecomposable modules $R_{w}$ are perhaps most natural from the perspective of geometry or representation theory, Definition 1 (and the study of knot homology, which we discuss briefly in Section 2) encourages us to concentrate on the tensor product modules $R_{\mathbf{i}}$. We call these particular Soergel bimodules Bott-Samelson for reasons which will be clarified in Section 5. 
Bott-Samelson bimodules are naturally identified with the equivariant cohomology of the "groupy" Bott-Samelson space

$$
G_{\mathbf{i}} \cong P_{s} \times_{B} P_{t} \times_{B} \cdots \times_{B} P_{u},
$$

where $P_{S}$ is the parabolic associated to the reflection $s$. The analogues of theorems connecting the $B \times B$-orbit closures in $G$ with Soergel bimodules are true here.

Theorem 1.6 If $G=\operatorname{SL}(n)$ or $\operatorname{GL}(n)$, then for all $\mathbf{i}$, we have

$$
R_{\mathbf{i}} \cong H_{B \times B}^{*}\left(G_{\mathbf{i}}\right) \quad H H_{*}\left(R_{\mathbf{i}}\right) \cong H_{B}^{*}\left(G_{\mathbf{i}}\right) .
$$

The $T$-conjugation on $G_{\mathbf{i}}$ is equivariantly formal, and the injection

$$
i_{T}^{*}: H_{T}^{*}\left(G_{\mathbf{i}}\right) \rightarrow H_{T}^{*}\left(G_{\mathbf{i}}^{T}\right)
$$

induces a bigrading on $H_{T}^{*}\left(G_{\mathbf{i}}\right)$ matching that on $H H_{*}\left(R_{\mathbf{i}}\right)$ as in Theorem 1.4.

The structure of the paper is as follows: In Section 2, we discuss the importance of the Hochschild homology of Soergel bimodules in knot theory. In Section 4, we prove Theorem 1.2, using the formalism of dg-modules, the relevant points of which we will summarize in Section 3. In Section 5, we will cover in more detail how to construct Soergel bimodules as equivariant intersection cohomology of various varieties. Finally, in Section 6, we prove Theorems 1.3-1.6.

Acknowledgments The authors would like to thank Catharina Stroppel, Olaf Schnürer, Allen Knutson, Shrawan Kumar, Peter Fiebig, Michel Brion and Tom Baird for their helpful suggestions, and Wolfgang Soergel for discussions which greatly enhanced their understanding of the equivariant derived category.

They would also like to thank the organizers of the conference "Microlocal and Geometric Methods in Representation Theory" and the staff of Schloss Reisensburg, where the authors first began their collaboration, as well as Nicolai Reshetikhin, Henning Haahr Andersen and Jørgen Ellegaard Andersen for making possible their visits to Århus, where this work progressed to its current state.

BW was supported under an NSF Graduate Fellowship, an NSF Postdoctoral Fellowship, a Clay Liftoff Fellowship, the RTG grant DMS-0354321, and a Danish National Research Foundation Niels Bohr Professorship grant.

GW was supported by an Eleanor Sophia Wood traveling scholarship and a Liegrits predoc scholarship. This paper forms part of the GW's PhD thesis. 


\section{Knot homology and Soergel bimodules}

While Soergel bimodules merit study on the basis of their connections to representation theory and geometry, we also have applications in knot theory in mind, as we will now briefly describe. The interested reader can find more details in the papers of Khovanov [11], Rasmussen [12] and Webster [16].

The braid group $B_{G}$ of $G$ is a finitely presented group, with generators $\sigma_{s}$ for each simple reflection $s \in W$, which is defined by the presentation:

$$
\begin{aligned}
\sigma_{s} \sigma_{t} & =\sigma_{t} \sigma_{s} & & \left(\text { when }(s t)^{2}=e\right) \\
\sigma_{s} \sigma_{t} \sigma_{s} & =\sigma_{t} \sigma_{s} \sigma_{t} & & \left(\text { when }(s t)^{3}=e\right) \\
\left(\sigma_{s} \sigma_{t}\right)^{2} & =\left(\sigma_{t} \sigma_{s}\right)^{2} & & \left(\text { when }(s t)^{4}=e\right) \\
\left(\sigma_{s} \sigma_{t}\right)^{3} & =\left(\sigma_{t} \sigma_{s}\right)^{3} & & \left(\text { when }(s t)^{6}=e\right)
\end{aligned}
$$

Note that if $G=\operatorname{SL}(n)$, then $B_{n}=B_{G}$ is the standard braid group familiar from knot theory.

There are several natural weak actions of the braid group on category $\mathcal{O}$ by families of functors (see, for example, Andersen and Stroppel [2] and Khomenko and Mazorchuk [9]), which have an avatar on the bimodule side of the picture in the form of a complex of bimodules attached to each braid group element. The description of these bimodule complexes can be found in various sources, for example Khovanov [11], or for general Coxeter groups in Rouquier [13].

Define the complexes of $S$-bimodules:

$$
\begin{aligned}
F\left(\sigma_{s}\right) & =\cdots \longrightarrow S[-1] \longrightarrow R_{S}[1] \longrightarrow 0 \longrightarrow \cdots \\
F\left(\sigma_{s}^{-1}\right) & =\cdots \longrightarrow 0 \longrightarrow R_{S}[1] \longrightarrow S[1] \longrightarrow \cdots
\end{aligned}
$$

where the maps between nonzero spaces are the unique (up to scalar) nonzero maps of degree 0 . These maps are defined by (respectively) the pushforward and pullback in $B \times B$-equivariant cohomology for the inclusion $B \hookrightarrow \overline{B s B}$.

Theorem 2.1 The shuffling complex

$$
F(\sigma) \stackrel{\text { def }}{=} F\left(\sigma_{i_{1}}^{\epsilon_{1}}\right) \otimes_{S} \cdots \otimes_{S} F\left(\sigma_{i_{m}}^{\epsilon_{m}}\right)
$$

of a braid $\sigma=\sigma_{i_{1}}^{\epsilon_{1}} \cdots \sigma_{i_{m}}^{\epsilon_{m}}$ (where $\epsilon_{i}= \pm 1$ ) depends up to homotopy equivalence only on $\sigma$, and not on its factorization. In particular,

$$
F\left(\sigma \sigma^{\prime}\right) \cong F(\sigma) \otimes_{S} F\left(\sigma^{\prime}\right),
$$

so $F$ defines a categorification of $B_{G}$. 
The maps in this complex also have a geometric interpretation: each degree is a direct sum of Bott-Samelson modules for subsequences of $\mathbf{i}$, and the "matrix coefficients" of the differential between these are induced by pullback or pushforward maps on $B \times B$-equivariant cohomology for inclusions $G_{\mathrm{i}^{\prime}} \rightarrow G_{\mathrm{i}^{\prime \prime}}$ of Bott-Samelson spaces where $\mathbf{i}^{\prime}$ and $\mathbf{i}^{\prime \prime}$ are subsequences of $\mathbf{i}$ which differ by a single index.

Even better, this complex can be used to define a knot invariant, as was shown by Khovanov [11]. Given an $S$-bimodule $R$ let $H H_{*}(R)$ be the Hochschild homology of $R$, which can be defined (using the standard equivalence between $S$-bimodules and $S \otimes S^{\text {op }}$-modules) by

$$
H H_{i}(R)=\operatorname{Tor}_{S \otimes S^{\mathrm{op}}}^{i}(S, R) .
$$

This can be calculated by the Hochschild complex of $S$ (which is often used as a definition), or by the Koszul complex, both of which are free resolutions of $S$ as an $S$-bimodule (that is, as an $S \otimes S^{\text {op }}$-module).

In the case where $G=\operatorname{SL}(n)$, Hochschild homology is a categorification of the trace on the braid group defined by Jones [8]. Remarkably, combining these creates a categorification of knot polynomials. The categorification obtained in this way had previously been defined by Khovanov and Rozansky.

Theorem 2.2 (Khovanov [11]) As a graded vector space, the homology $\mathcal{K} \mathcal{R}(\bar{\sigma})$ of the complex $H H_{i}(F(\sigma))$ depends (after appropriate grading shift) only on the knot $\bar{\sigma}$ formed by closing $\sigma$ and is in fact the triply graded homology defined by Khovanov and Rozansky in [10].

Combining Theorem 1.2 and our remarks above, we can understand the differentials of the complex $H H_{i}(F(\sigma))$ in terms of pullback and pushforward on $B$-equivariant cohomology.

\section{The equivariant derived category and dg-modules}

Since our readers may not be well-acquainted with the formalism of equivariant derived categories and its connection with dg-modules, as developed by Bernstein and Lunts, we will provide a brief overview of the necessary background. This material is discussed in more detail and greater generality in their monograph [4].

We will begin with some motivation. Suppose a Lie group $G$ operates on a space $X$. We have maps:

$$
\begin{array}{ll}
m: G \times X \rightarrow X & m(g, x)=g \cdot x \\
\pi: G \times X \rightarrow X & \pi(g, x)=x
\end{array}
$$


A function $f$ on $X$ is $G$-invariant if and only if $m^{*} f=\pi^{*} f$. It is therefore natural to define a $G$-equivariant sheaf on $X$ to be a sheaf $\mathcal{F}$ on $X$ together with an isomorphism $\theta: m^{*} \mathcal{F} \rightarrow \pi^{*} \mathcal{F}$. (There is also a cocycle condition that we don't mention here).

One can show that if $G$ operates topologically freely on $X$ with quotient $X / G$ then the categories of $G$-equivariant sheaves on $X$ and sheaves on $X / G$ are equivalent.

Faced with a $G$-space, one would like to define an "equivariant derived category". This should associate to a pair $(G, X)$ a triangulated category $D_{G}^{b}(X)$ together with a "forgetting $G$-equivariance" functor $D_{G}^{b}(X) \rightarrow D^{b}(X)$. For any reasonable definition of $D_{G}^{b}(X)$, whenever $G$ acts topologically freely, there should be a natural equivalence $D_{G}^{b}(X) \cong D^{b}(X / G)$, as well as notions of pullback and pushforward for equivariant maps.

The trick is to notice that, at least up to homotopy, we may assume that the action is free: we "liberate" $X$ (ie make it free) by replacing it with $X \times E G$ where $E G$ is the total space of the universal $G$-bundle (ie any contractible space on which $G$ acts topologically freely). The first projection $p: X \times E G \rightarrow X$ is a homotopy equivalence (because $E G$ is contractible) and the diagonal operation of $G$ on $X \times E G$ is free. Thus, we can consider the quotient map $q: X \times E G \rightarrow X \times \times_{G} E G$ as the "liberation" of $X \rightarrow X / G$.

We will now try to make this more precise. For the rest of this section, assume that $G$ is a connected complex linear algebraic group and $X$ a complex $G$-variety (both equipped with the classical topology). There exists an $G$-space $E G$ such that:

(1) $G$ acts topologically freely on $E G$,

(2) $E G$ is contractible,

(3) we may write $E G$ as a direct limit of smooth complex $G$-varieties $E_{n} G$, where each inclusion $E_{n} G \hookrightarrow E_{n+1} G$ is a $G$-equivariant closed immersion.

For example, we may embed $G$ in some $G L(n)$ and then take a direct limit of Stiefel manifolds. We let $X \times{ }_{G} E G$ denote the quotient of $X \times E G$ by the diagonal action. In the case where $X$ is a single point, we have $X \times_{G} E G \cong E G / G$, which we will denote by $B G$. Given a space $Y$, we denote by $D^{b}(Y)$ the bounded derived category of sheaves of $\mathbb{C}$-vector spaces on $Y$.

Definition 2 The (bounded) equivariant derived category $D_{G}^{b}(X)$ is the full subcategory of $D^{b}\left(X \times{ }_{G} E G\right)$ consisting of complexes $\mathcal{F} \in D^{b}\left(X \times_{G} E G\right)$ such that $q^{*} \mathcal{F} \cong p^{*} \mathcal{G}$ for some complex $\mathcal{G} \in D^{b}(X)$. 
Remark 1 This is not exactly Bernstein and Lunts' definition. Consider the following diagram of spaces:

$$
X \stackrel{p}{\leftarrow} X \times E G \stackrel{q}{\rightarrow} X \times_{G} E G .
$$

They construct a category $D_{G}^{b}(X)^{\mathrm{BL}}$ as follows: objects are tuples $(\mathcal{G}, \mathcal{F}, \alpha)$ where $\mathcal{G} \in D^{b}(X), \mathcal{F} \in D^{b}\left(X \times_{G} E G\right)$, and $\alpha: q^{*} \mathcal{F} \rightarrow p^{*} \mathcal{G}$ is an isomorphism; and morphisms between two objects $(\mathcal{G}, \mathcal{F}, \alpha)$ and $\left(\mathcal{G}^{\prime}, \mathcal{F}^{\prime}, \alpha^{\prime}\right)$ consist of pairs $(g, f)$ where $g: \mathcal{G} \rightarrow \mathcal{G}^{\prime}$ and $f: \mathcal{F} \rightarrow \mathcal{F}^{\prime}$ are morphisms such that the diagram

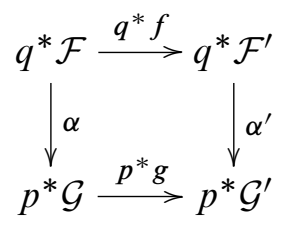

commutes. One may check that the functor $D_{G}^{b}(X)^{\mathrm{BL}} \rightarrow D_{G}^{b}(X)$ which maps an object $(\mathcal{G}, \mathcal{F}, \alpha)$ to $\mathcal{F}$ and a morphism $(g, f)$ to $f$ is an equivalence of categories.

Remark 2 The space $X \times_{G} E G$ is usually infinite dimensional. However, one may choose the spaces $E_{n} G$ such that the quotient $X \times_{G} E_{n} G$ is an algebraic variety. In this case $D_{G}^{b}(X)$ has an alternative definition as a certain limit of subcategories in $D^{b}\left(X \times_{G} E_{n} G\right)$ (see Bernstein and Lunts [4]).

As previously mentioned, it is natural to expect that there will exist a "forgetting $G$-equivariance functor" For: $D_{G}^{b}(X) \rightarrow D^{b}(X)$. With $p$ and $q$ as in the Remark 1 , one defines $\operatorname{For}(\mathcal{F})=p_{*} q^{*} \mathcal{F}$.

If $H \subset G$ is a subgroup, then $H$ acts topologically freely on $E G$, so we may take $E G$ for $E H$. Thus, we have a natural map

$$
\varphi_{H}^{G}: X \times_{H} E G \rightarrow X \times_{G} E G
$$

commuting with the projection to $X$. The pullback and pushforward by this map induce functors

$$
\left(\varphi_{H}^{G}\right)^{*}=\operatorname{res}_{H}^{G}: D_{G}^{b}(X) \rightarrow D_{H}^{b}(X), \quad\left(\varphi_{H}^{G}\right)_{*}=\operatorname{ind}_{G}^{H}: D_{H}^{b}(X) \rightarrow D_{G}^{b}(X) .
$$

Similarly, for any $G$-space we have a map $X \times{ }_{G} E G \rightarrow B G$. Because $X$ has finite dimensional cohomology, pushforward yields a functor

$$
\pi_{*}: D_{G}^{b}(X) \rightarrow D_{G}^{b}(\mathrm{pt})
$$

which commutes with the induction and restriction functors. 
In this work, we are interested in equivariant cohomology for connected complex linear algebraic groups. This emerges as the cohomology of objects living in $D_{G}(\mathrm{pt})$. The first key observation of Bernstein and Lunts is the following:

Proposition 3.1 If $G$ is a connected complex algebraic group, then $D_{G}^{b}(\mathrm{pt})$ is the full triangulated subcategory of $D^{b}(B G)$ generated by the constant sheaf.

It turns out that this observation allows Bernstein and Lunts to give an algebraic description of $D_{G}(\mathrm{pt})$. For this we need the language of differential graded algebras and modules.

Definition 3 A differential graded algebra (or dg-algebra) is a unital, graded associative algebra $\mathcal{A}=\oplus_{i \in \mathbb{Z}} A_{i}$ together with an additive endomorphism $d: \mathcal{A} \rightarrow \mathcal{A}$ of degree 1 such that:

(1) $d$ is a differential: ie $d^{2}=0$,

(2) $d$ satisfies the Leibniz rule: $d(a b)=(d a) b+(-1)^{\operatorname{deg} a} a(d b)$,

(3) $d\left(1_{\mathcal{A}}\right)=0$, where $1_{\mathcal{A}}$ denotes the identity of $\mathcal{A}$.

A left differential graded module (or left dg-module) over a differential graded algebra $\mathcal{A}$ is a graded left $\mathcal{A}$-module $M$ together with a differential $d_{M}: M \rightarrow M$ of degree 1 satisfying:

(1) $d_{M}^{2}=0$,

(2) $d_{M}(a m)=(d a) m+(-1)^{\operatorname{deg} a} a\left(d_{M} m\right)$ for $a \in \mathcal{A}$ and $m \in M$.

A morphism of dg-modules is a graded $\mathcal{A}$-module homomorphism $f: M \rightarrow M^{\prime}$ commuting with the differentials.

Remark 3 If $\mathcal{A}=A_{0}$ is concentrated in degree zero, then a differential graded module is just a chain complex of $\mathcal{A}$-modules.

Given any dg-module $M$, we may consider its cohomology $H^{*}(M)$, which is a graded module over the graded algebra $H^{*}(\mathcal{A})$. As with the category of modules over an algebra, the category of dg-modules over a dg-algebra has a homotopy category and a derived category, as defined by Bernstein and Lunts [4]. A map $f: M \rightarrow M^{\prime}$ of dg-modules is a quasi-isomorphism if the induced map $H^{*}(M) \rightarrow H^{*}\left(M^{\prime}\right)$ on cohomology is an isomorphism. After localizing quasi-isomorphisms, one obtains the derived category of dg-modules for the $\operatorname{dg}$-algebra $\mathcal{A}$, which we denote by $\operatorname{dg} \operatorname{Mod} \mathcal{A}$. 
We denote by $\operatorname{dgMod}^{f} \mathcal{A}$ the full subcategory consisting of objects isomorphic to dg-modules which are finitely generated over $\mathcal{A}$.

Given a morphism $\mathcal{A} \rightarrow \mathcal{A}^{\prime}$ of dg-algebras, we would like to define functors of restriction and extension of scalars between the categories $\operatorname{dg} \operatorname{Mod} \mathcal{A}$ and $\operatorname{dg} \operatorname{Mod} \mathcal{A}^{\prime}$. Restriction of scalars is unproblematic (acyclic complexes are mapped to acyclic complexes) but more care is needed in defining extension of scalars. Just as in the normal derived category, one needs a special class of objects in order to define functors. $\operatorname{In} \operatorname{dg} \operatorname{Mod} \mathcal{A}$ these are the $\mathcal{K}$-projective objects [4], which we will not discuss in complete generality. In the sequel, we will only be interested in a special class of dg-algebras in which it is possible to construct $\mathcal{K}$-projective objects rather explicitly.

Proposition 3.2 $\left[4\right.$, Proposition 11.1.1] Let $\mathcal{A}=\mathbb{C}\left[x_{1}, \ldots x_{n}\right]$ be viewed as a $d g$ algebra by setting $d_{\mathcal{A}}=0$ and requiring that each $x_{i}$ have even degree. Then all $d g$-modules which are free as $\mathcal{A}$-modules are $\mathcal{K}$-projective.

As it will be important for later arguments, we will describe how to construct a $\mathcal{K}-$ projective resolution of a dg-module $M$ when $\mathcal{A}$ is as in the proposition, and $M$ has trivial differential. We may choose a free resolution in the category of graded $\mathcal{A}$-modules:

$$
P_{-n} \rightarrow \cdots \rightarrow P_{-2} \rightarrow P_{-1} \rightarrow M
$$

We then consider the direct sum $P=\oplus_{i} P_{-i}[i]$ as a dg-module which carries a natural differential: elements in $P_{-i}[i]$ are mapped under $d_{P}$ into $P_{-i+1}[i-1]$ using the corresponding map in the above resolution. The natural morphism $P \rightarrow M$ (again induced from the resolution above) is a quasi-isomorphism.

Using standard techniques from homological algebra, one may construct $\mathcal{K}$-projective resolutions for dg-modules over $\mathcal{A}$ with nontrivial differential, but this will not be necessary for our results.

We may now define the extension of scalars functor. Suppose we have a morphism $\mathcal{A} \rightarrow \mathcal{A}^{\prime}$ of dg-algebras, and that $\mathcal{A}$ and $\mathcal{A}^{\prime}$ are as in the proposition. For any $N \in$ $\operatorname{dg} \operatorname{Mod} \mathcal{A}$, we define extension of scalars by

$$
\mathcal{A}^{\prime} \stackrel{L}{\otimes_{\mathcal{A}}} N \stackrel{\text { def }}{=} \mathcal{A}^{\prime} \otimes_{\mathcal{A}} P
$$

where $P$ is a $\mathcal{K}$-projective resolution of $P$ and the differential on the later module is given by $d(a \otimes p)=a \otimes d_{P}(p)$ for $a \in \mathcal{A}^{\prime}$ and $p \in P$. (Alternatively we may also take a $\mathcal{K}$-projective resolution of $\mathcal{A}^{\prime}$ as an $\mathcal{A}$ dg-module).

We can now return to a discussion of the equivariant derived category. Abelian and triangulated categories can often be described by "module categories" over endomorphism 
rings of generators. We have already seen that $D_{G}^{b}(\mathrm{pt})$ is the triangulated subcategory of $D^{b}(B G)$ generated by the constant sheaf. Hence this category should be described in terms of the endomorphisms of $\underline{\mathbb{C}}_{B G}$ in the derived category (which is naturally a dg-algebra).

First, we must describe this algebra: there is a quasi-isomorphism

$$
\mathcal{A}_{G} \stackrel{\text { def }}{=} H^{*}(B G) \rightarrow \operatorname{End}_{D^{b}(B G)}^{\bullet}\left(\mathbb{C}_{B G}\right)
$$

of dg-algebras. This yields a functor

$$
\Gamma_{G}=\operatorname{Hom}_{D^{b}(B G)}^{\bullet}\left(\mathbb{C}_{B G},-\right): D_{G}^{b}(\mathrm{pt}) \rightarrow \operatorname{dgMod}^{f} \mathcal{A}_{G} .
$$

Bernstein and Lunts then show:

Theorem 3.3 (Main Theorem of Bernstein-Lunts [4]) Assume as before that $G$ is a connected complex algebraic group. The above functor gives an equivalence of triangulated categories commuting with the cohomology functor:

$$
\Gamma_{G}: D_{G}^{b}(\mathrm{pt}) \rightarrow \operatorname{dgMod}^{f} \mathcal{A}_{G}
$$

Moreover if $\varphi: G \rightarrow H$ is an inclusion and $\mathcal{A}_{H} \rightarrow \mathcal{A}_{G}$ is the induced homomorphism, then the restriction and induction functors have an algebraic description in terms of dg-modules:
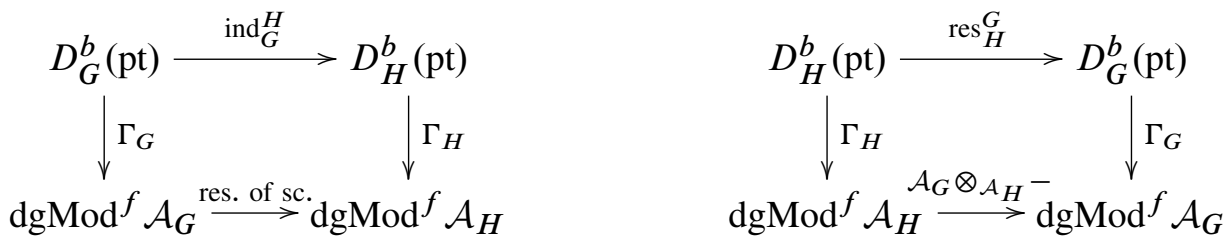

Remark 4 Note that if $G$ is a connected complex linear algebraic group, then $\mathcal{A}_{G}$ is always a polynomial ring on even generators. In this case, by Proposition 3.2 and the above discussion, we can describe the $\mathcal{K}$-projective objects and hence compute the effect of the restriction and induction functors in terms of dg-modules.

We will now describe equivariant intersection cohomology complexes, which will be important in the sequel. Given a complex variety $X$ and a smooth locally closed subvariety $U \subset X$, there is a complex $\mathbf{I C}(U) \in D^{b}(X)$ called the intersection cohomology complex, with remarkable properties (see for example Beilinson, Bernstein and Deligne [3] and Goresky and MacPherson [7]). We normalize $\mathbf{I C}(U)$ so that, if $j: U \hookrightarrow X$ denotes the inclusion, then

$$
j^{*} \mathbf{I C}(U) \cong \underline{\mathbb{C}}_{U}
$$


where $\mathbb{C}_{U}$ denotes the constant sheaf on $U$ in degree 0 . If $U \subset X$ is in addition dense, we may define the intersection cohomology of $X$, denoted $I H^{*}(X)$, to be the hypercohomology of $\mathbf{I C}(U)$. In other words

$$
I H^{*}(X) \stackrel{\text { def }}{=} \mathbb{H}^{*}(\mathbf{I C}(U)) .
$$

It does not depend on the choice of the dense smooth subvariety $U \subset X$.

It is possible to construct equivariant analogues of the intersection cohomology complexes, as described in Chapter 5 of [4]: If $X$ is furthermore a $G$-variety for a complex algebraic group $G$ and $U$ is a smooth $G$-stable subvariety, there exists an equivariant intersection cohomology complex which we denote by $\mathbf{I C}_{G}(U)$. One then defines the equivariant intersection cohomology by

$$
I H_{G}^{*}(X) \stackrel{\text { def }}{=} \mathbb{H}^{*}\left(\mathbf{I C}_{G}(U)\right) .
$$

where $U \subset X$ is dense. The complex $\operatorname{IC}_{G}(U)$ is determined up to isomorphism in $D_{G}^{b}(X)$ by requiring that $\operatorname{For}\left(\mathbf{I C}_{G}(U)\right) \cong \mathbf{I C}(U)$. It follows that equivariant intersection cohomology complexes behave well with respect to restriction:

Lemma 3.4 If $H \hookrightarrow G$ is an inclusion of complex linear algebraic groups, $X$ is a $G$-variety and $U$ is a smooth $G$-stable subvariety then:

$$
\operatorname{res}_{G}^{H} \mathbf{I C}_{G}(U) \cong \mathbf{I C}_{H}(U)
$$

Remark 5 In dealing with equivariant intersection cohomology complexes, it is more convenient to work with the equivalent definition of the equivariant derived category mentioned in Remark 2.

\section{Hochschild homology and dg-algebras}

Recall that the Hochschild homology of an $S$-bimodule $R$ can be defined as

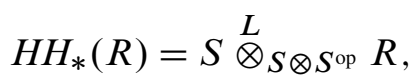

where $S$ has been made into an $S \otimes S^{\text {op }}$ algebra by left and right multiplication. Since $S \cong \mathcal{A}_{B}$ and $S \otimes S \cong \mathcal{A}_{B \times B}$, this map is that induced by the diagonal group homomorphism $B \hookrightarrow B \times B$. Thus, we expect that the geometric analogue of taking Hochschild homology is restricting from a $B \times B$-action to the diagonal $B$.

However, we must be careful about the difference between dg-modules and modules. Hochschild homology is an operation on $S$-bimodules, not dg-bimodules. Thus, to make a precise statement requires us to restrict to formal complexes. 
Definition 4 Let $M \in \operatorname{dgMod} \mathcal{A}_{G}$ be a dg-module. If $M \cong H^{*}(M)$ in $\operatorname{dgMod} \mathcal{A}_{G}$ we say that $M$ is formal. Similarly, $\mathcal{F} \in D_{G}(\mathrm{pt})$ is formal if $\Gamma_{G}(\mathcal{F})$ is.

The following proposition connects the Hochschild cohomology of formal equivariant sheaves with another equivariant cohomology. This is our main technical tool.

Proposition 4.1 Suppose $\mathcal{F} \in D_{B \times B}(\mathrm{pt})$ is formal. Then one has an isomorphism:

$$
\bigoplus_{i} H H_{i}\left(\mathbb{H}_{B \times B}^{*}(\mathcal{F})\right)[i] \cong \mathbb{H}_{B}^{*}\left(\operatorname{res}_{B \times B}^{B} \mathcal{F}\right)
$$

as graded $S$-modules.

Furthermore this isomorphism is functorial. That is, if $\mathcal{F}$ and $\mathcal{G}$ are formal sheaves in $D_{B \times B}(\mathrm{pt})$, and $\varphi: \mathcal{F} \rightarrow \mathcal{G}$ is a morphism, the maps $\mathbb{H}_{B}^{*}(\varphi)$ and $H H_{*}\left(\mathbb{H}_{B \times B}^{*}(\varphi)\right)$ commute with this isomorphism.

Proof of Proposition 4.1 In order to work out the Hochschild homology of $\mathbb{H}_{B \times B}^{*}(\mathcal{F})$ we may take a free resolution of $\mathbb{H}_{B \times B}^{*}(\mathcal{F})$ by $S \otimes S$-modules:

$$
0 \rightarrow P_{-2 n} \rightarrow \cdots \rightarrow P_{-1} \rightarrow \mathbb{H}_{B \times B}^{*}(\mathcal{F})
$$

We then apply $S \otimes_{S \otimes S}-$ and take cohomology. However, because $\mathbb{H}_{B \times B}^{*}(\mathcal{F}) \cong$ $\Gamma_{B \times B}(\mathcal{F})$ in $\operatorname{dgMod} \mathcal{A}_{B \times B}$, we may also regard $\bigoplus P_{i}[-i]$ as a $\mathcal{K}$-projective resolution of $\Gamma(\mathcal{F})$. By Theorem 3.3 we have:

$$
\mathbb{H}_{B}^{*}\left(\operatorname{res}_{B \times B}^{B} \mathcal{F}\right) \cong H^{*}\left(S \stackrel{L}{\otimes}{ }_{S \otimes S} \mathbb{H}_{B \times B}^{*}(\mathcal{F})\right) \cong \bigoplus H H_{i}\left(\mathbb{H}_{B \times B}^{*}(\mathcal{F})\right)[i]
$$

Proof of Theorem 1.2 Let $\mathcal{F}$ denote the image of the intersection cohomology sheaf on $\overline{B w B}$ in $D_{B \times B}(\mathrm{pt})$. By Proposition $5.2, \mathcal{F}$ is formal. Hence we can apply Proposition 4.1. However, we also know that $\mathbb{H}_{B \times B}^{*}(\mathcal{F})$ is the indecomposable Soergel bimodule $R_{w}$. Thus:

$$
\bigoplus_{i} H H_{i}\left(R_{w}\right)[i] \cong \mathbb{H}_{B}^{*}\left(\operatorname{res}_{B \times B}^{B} \mathcal{F}\right)
$$

But $\operatorname{res}_{B \times B}^{B}$ commutes with the map to a point and by Lemma 3.4,

$$
\operatorname{res}_{B \times B}^{B}\left(\mathbf{I C}_{B \times B}(B w B)\right) \cong \mathbf{I C}_{B}(B w B)
$$

Therefore

$$
\bigoplus_{i} H H_{i}\left(R_{w}\right)[i] \cong \mathbb{H}_{B}^{*}\left(\mathbf{I C}_{B}(B w B)\right) \cong I H_{B}^{*}\left(G_{w}\right)
$$

which yields the main theorem. 


\section{The geometry of Bott-Samelson bimodules}

In this section, we discuss Bott-Samelson bimodules, calculate their $B \times B$-equivariant cohomology and obtain the formality results needed in the previous section.

Since we have already described the geometric realization of indecomposable bimodules as intersection cohomology of subvarieties of $G$, we know abstractly that the BottSamelson bimodule $R_{\mathbf{i}}$ must be the hypercohomology of a perverse sheaf obtained by taking a direct sum of IC-sheaves of these subvarieties with appropriate multiplicities.

However, this is deeply dissatisfying from a geometric viewpoint, and totally at odds with our viewpoint that Bott-Samelson bimodules are very natural objects. Thus we would like a more natural geometric realization of them.

For each simple reflection $s$, let $P_{s}$ be the minimal parabolic containing $s$. For a sequence $\mathbf{i}=(s, t, \ldots, u)$ of simple reflections, let

$$
G_{\mathbf{i}}=P_{s} \times{ }_{B} P_{t} \ldots \cdots \times{ }_{B} P_{u} .
$$

We call this the Bott-Samelson variety corresponding to $\mathbf{i}$. Note that this variety still carries a $B \times B$-action, and thus a diagonal $B$-action.

Furthermore, we have a projective $B \times B$-equivariant map $m_{\mathbf{i}}$ : $G_{\mathbf{i}} \rightarrow G$ given by multiplication, which intertwines the diagonal $B$-action on $G_{\mathbf{i}}$ with the conjugation $B$-action on $G$.

The quotient of $G_{\mathbf{i}}$ by the right Borel action is the familiar projective Bott-Samelson variety which is used to construct resolutions of singularities for Schubert varieties. It is worth noting that just like in the flag variety case, if $\mathbf{i}$ is a reduced expression (ie if $\ell(s t \ldots u)$ is the length of $\mathbf{i})$, then the multiplication map is a resolution of singularities.

Let us explain how to calculate the $B \times B$-equivariant cohomology of the Bott-Samelson varieties. Actually, we will calculate the corresponding dg-module over $S \otimes S$. We start with a lemma:

Lemma 5.1 Suppose $\mathcal{F} \in D_{B \times B}^{b}(\mathrm{pt})$ is formal and let $s$ be a simple reflection. Then

$$
\Gamma_{B \times B}\left(\operatorname{res}_{P_{S} \times B}^{B \times B} \operatorname{ind}_{B \times B}^{P_{s} \times B} \mathcal{F}\right) \cong S \otimes S^{s} \Gamma_{B \times B}(\mathcal{F})
$$

as objects in $\operatorname{dgMod}_{\mathcal{A}_{B \times B}}^{f}$.

Proof Thanks to Theorem 3.3 we know that $\Gamma_{P_{s} \times B}\left(\operatorname{ind}_{B \times B}^{P_{s} \times B} \mathcal{F}\right)$ is equal to $\Gamma_{B \times B}(\mathcal{F})$ regarded as dg-module over $S^{s} \otimes S$. Hence

$$
\Gamma_{B \times B}\left(\operatorname{res}_{P_{S} \times B}^{B \times B} \operatorname{ind}_{B \times B}^{P_{S} \times B} \mathcal{F}\right)=(S \otimes S) \stackrel{L}{\otimes} S_{S}^{s} \otimes S \Gamma_{B \times B}(\mathcal{F}) .
$$


However, $S \otimes S$ is free as a module over $S^{S} \otimes S$ and is hence $\mathcal{K}$-projective. Thus the derived tensor product coincides with the naive tensor product and the result follows.

We can now prove the crucial "formality" claim mentioned above:

Proposition 5.2 The direct images of the sheaves $\underline{\mathbb{C}}_{G_{\mathrm{i}}}$ and $\mathbf{I C}_{B \times B}(B w B)$ in $D_{B \times B}^{b}(\mathrm{pt})$ are formal.

Proof First notice that we can write the sheaf $\left(m_{\mathbf{i}}\right)_{*} \underline{\mathbb{C}}_{G_{\mathrm{i}}}$ as an iterated induction and restriction:

$$
\left(m_{\mathrm{i}}\right)_{*} \underline{\mathbb{C}}_{G_{\mathrm{i}}} \cong \operatorname{res}_{P_{s} \times B}^{B \times B} \operatorname{ind}_{B \times B}^{P_{s} \times B} \cdots \operatorname{res}_{P_{u} \times B}^{B \times B} \operatorname{ind}_{B \times B}^{P_{u} \times B}\left(\underline{\mathbb{C}}_{B}\right) .
$$

Hence by the above lemma, letting $p$ be the projection to a point:

$$
\Gamma_{B \times B}\left(p_{*} \underline{\mathbb{C}}_{G_{\mathbf{i}}}\right)=S \otimes S^{s} S \otimes_{S^{t}} \otimes \cdots \otimes_{S^{u}} S \quad \text { in } \operatorname{dgMod}_{S \otimes S}^{f} .
$$

Thus the proposition is true for $\mathbb{C}_{G_{\mathbf{i}}}$. Now, by the decomposition theorem of [3] (or more precisely, its equivariant version in [4]), we may obtain $\mathbf{I C}_{B \times B}(B w B)$ as a direct summand of $\left(m_{\mathbf{i}}\right)_{*} G_{\mathbf{i}}$, where $\mathbf{i}=(s, \ldots, u)$ is a reduced expression for $w$. Thus $p_{*} \mathbf{I C}_{B \times B}(B w B)$ is a direct summand of $p_{*} \underline{\mathbb{C}}_{G_{\mathrm{i}}}$ and is also formal.

\section{Equivariant formality}

Now, we will carry out some actual computations of $B$-equivariant cohomology, and thus of Hochschild homology.

Of course, the best setting in which to compute the equivariant cohomology of a variety is when that variety (or more precisely, the sheaf one intends to compute the hypercohomology of) is equivariantly formal.

Definition/Theorem 6.1 We call $\mathcal{F} \in D_{T}^{b}(X)$ on a $T$-variety $X$ equivariantly formal if one of the following equivalent conditions holds:

(1) The $S$ module $\mathbb{H}_{T}^{*}(X, \mathcal{F})$ is free.

(2) The differentials in the spectral sequence

$$
\mathbb{H}^{*}(X, \mathcal{F}) \otimes S \Rightarrow \mathbb{H}_{T}^{*}(X, \mathcal{F})
$$

are trivial, that is, if $\mathbb{H}^{*}(X, \mathcal{F}) \otimes S \cong \mathbb{H}_{T}^{*}(X, \mathcal{F})$ as $S$-modules.

(3) We have the equality $\operatorname{dim}_{\mathbb{C}} H^{*}(X)=\operatorname{dim}_{\mathbb{C}} H^{*}\left(X^{T}\right)$. 
The equivariant formality of the Bott-Samelsons of $\operatorname{SL}(n)$ has been proven by Rasmussen in different language.

Proposition 6.2 If $G=\operatorname{SL}(n)$ or $\operatorname{GL}(n)$, the $T$-space $G_{\mathbf{i}}$ is equivariantly formal for all $\mathbf{i}$.

Proof By Theorem 1.2, $H H_{*}\left(R_{\mathbf{i}}\right)$ is free as an $S$-module if and only if $H_{T}^{*}\left(G_{\mathbf{i}}\right)$ is. By [12, Propositon 4.6], the module $H H_{*}\left(R_{\mathbf{i}}\right)$ is free in type $A$, so by Definition/Theorem 6.1 above $G_{\mathbf{i}}$ is equivariantly formal.

This in turn implies that $m_{*} \underline{\mathbb{C}}_{G_{\mathbf{i}}}$ is equivariantly formal, where $m: G_{\mathbf{i}} \rightarrow G$ is the multiplication map. Since all summands of equivariant formal sheaves are themselves equivariantly formal, and each $\mathbf{I C}_{B \times B}(B w B)$ appears as a summand of such a sheaf (if, for example, $\mathbf{i}$ is a reduced word for $w$ ), this completes the proof of Theorem 1.3 and the first part of Theorem 1.6.

While the most obvious consequence of equivariant formality, calculating the equivariant cohomology from the ordinary cohomology or vice versa, is a useful one, there are less obvious ones as well.

Proposition 6.3 (Goresky, Kottwitz and MacPherson [6, Theorem 6.3]) If $\mathcal{F}$ is equivariantly formal, and $i: X^{T} \rightarrow X$ is the natural inclusion, then the pullback map

$$
i_{T}^{*}: H_{T}^{*}(X, \mathcal{F}) \rightarrow H_{T}^{*}\left(X^{T}, i^{*} \mathcal{F}\right)
$$

is injective.

As we mentioned earlier, we are interested in the Hochschild homology of Soergel bimodules as a bigraded object (so that we get a triply-graded knot homology theory), but the grading on equivariant hypercohomology is only one of these. From now on, we consider $H_{T}^{*}\left(G_{w}\right)$ as a bigraded $S$-module, with the bigrading defined by the isomorphism with Hochschild homology given by Proposition 4.1.

Proof of Theorem 1.4 and Theorem 1.6 Since the pullback map $H_{T}^{*}\left(G_{\mathbf{i}}\right) \rightarrow H_{T}^{*}\left(G_{\mathbf{i}}^{\boldsymbol{T}}\right)$ is induced by a map of Soergel bimodules, it is homogeneous in both gradings. Similar reasoning applies to the map induced by the inclusion of a summand $\mathbf{I C}_{B \times B}(B w B) \hookrightarrow$ $m_{*} \underline{\mathbb{C}}_{G_{\mathbf{i}}}$. Thus we need only establish the theorem for $G_{\mathbf{i}}^{T}$. As this is a union of complex tori with the trivial action, we need only establish the theorem for $T$.

This case follows directly from applying $H H_{0}$ to the Koszul resolution of $H_{T \times T}(T) \cong$ $S$ as a bimodule over itself. 
Let us turn to the case where $G_{w}$ is smooth. Since $H_{T}^{*}\left(G_{w}\right) \cong S \otimes H^{*}\left(G_{w}\right)$, we should address the structure of $H^{*}\left(G_{w}\right)$. Surprisingly, no description of this cohomology seems to be in the literature, but, in fact, there is a very beautiful one.

As is well known (and we reprove in the course of Lemma 6.6 below), there exists a unique decreasing sequence of positive integers $k_{1}, \cdots k_{n}$ such that the Hilbert series of $H^{*}\left(G_{w} / B\right)$ is of the form

$$
\sum_{i=1}^{\ell(w)} q^{i / 2} \operatorname{dim} H^{i}\left(G_{w} / B\right)=\prod_{j=1}^{n} \frac{1-q^{k_{j}}}{1-q} .
$$

When $w_{0}=w$, we have $G_{w}=G$, and the numbers $k_{i}$ are the exponents of the group $G$.

Theorem 6.4 If $G_{w}$ is smooth, then as an algebra

$$
H^{*}\left(G_{w}\right) \cong \wedge^{\bullet}\left(\gamma_{1}, \ldots \gamma_{n}\right),
$$

where $\operatorname{deg}\left(\gamma_{i}\right)=2 k_{i}-1$, and as an $S$-algebra

$$
H_{T}^{*}\left(G_{w}\right) \cong H H_{i}\left(R_{w}\right) \cong S \otimes H^{*}\left(G_{w}\right)
$$

In the standard double grading on $H H_{i}\left(R_{w}\right)$, we have $\operatorname{deg}\left(1 \otimes \gamma_{i}\right)=\left(1,2 k_{i}\right)$.

This immediately implies Theorem 1.5. Furthermore, this explicitly describes the Hilbert series of $H H_{i}\left(R_{w}\right)$, proving a conjecture of Rasmussen.

Corollary 6.5 The Hilbert series of $H H_{i}\left(R_{w}\right)$ is given by:

$$
\sum_{i, j} a^{i} q^{j} \operatorname{dim}_{\mathbb{C}} H H_{i}\left(R_{w}\right)_{2 j}=\prod_{\ell=1}^{n} \frac{1+a q^{k_{\ell}}}{1-q}
$$

Since $\operatorname{dim}_{\mathbb{C}} H^{*}\left(G_{w}\right)=2^{\mathrm{rk}(G)}$, Definition/Theorem 6.1(3) establishes the equivariant formality of $G_{w}$ independently of the earlier results of this paper (and for all types).

As usual in Lie theory, we define the height $h(\alpha)=\langle\rho, \alpha\rangle$ of a root $\alpha$ to be its evaluation against the sum of the fundamental weights, that is, the height is the linear extension of the function which assigns 1 to each simple root.

Let $\mathcal{R}_{+}$be the set of positive roots of $G$, and $\mathcal{R}_{-}$be the set of negative ones.

Lemma 6.6 The cohomology ring $H^{*}\left(G_{w} / B\right)$ is a quotient of the polynomial ring $S$ by a regular sequence $\left(f_{1}, \ldots, f_{n}\right)$. 
Proof By results of Akyıldiz and Carrell [1], the ring $H^{*}\left(G_{w} / B\right)$ is a quotient of $\mathbb{C}[B w B / B]$ by a regular sequence $\left(g_{1}, \ldots, g_{k}\right)$, where $k=\ell(w)$.

In this grading, $\mathbb{C}[B w B / B]$ is a polynomial algebra generated by elements of degree $2 h(\alpha)$ for each root $\alpha \in \mathcal{R}_{+} \cap w^{-1}\left(\mathcal{R}_{-}\right)$, and the degrees of $g_{i}$ are given by $2 h(\alpha)+2$ as $\alpha$ ranges over the same roots. Corresponding to the simple roots are $m$ generators $y_{1}, \ldots y_{m}$ of degree 2 , which are the first Chern classes of line bundles on $G / B$ corresponding the fundamental weights, and for the other roots we have $\ell(w)-m$ other generators $y_{m+1}, \ldots, y_{\ell}(w)$ of higher degree. Here, we assume these are in increasing order by degree.

It is a well known fact that the cohomology $\operatorname{ring} H^{*}(G / B)$ is generated by the Chern classes $x_{i}$. Since the natural pullback map $H^{*}(G / B) \rightarrow H^{*}\left(G_{w} / B\right)$ is onto, the ring $H^{*}\left(G_{w} / B\right)$ is also generated by the $x_{i}$, which span $H^{2}\left(G_{w} / B\right)$. That is, if $p=\operatorname{deg} y_{k}>2$, then for some $\beta_{i} \in \mathbb{C}$, we have the equality in $\mathbb{C}[B w B / B]$

$$
y_{k}-\sum_{\operatorname{deg}\left(g_{i}\right)=p} \beta_{i} g_{i} \in S+\sum_{\operatorname{deg}\left(g_{j}\right)<p} S g_{j} .
$$

Since $y_{k} \notin \sum_{\operatorname{deg}\left(g_{j}\right)<p} S g_{j}$, we can eliminate $y_{k}$ and any single relation $g_{i}$ such that $\beta_{i} \neq 0$. Obviously, $\left(g_{1}, \ldots, g_{n}\right) \backslash\left\{g_{i}\right\}$ is again a regular sequence in $\mathbb{C}\left[y_{1}, \ldots, y_{k-1}\right]$. Applying this argument inductively, we obtain a subsequence $\left(g_{i_{1}}, \ldots, g_{i_{n}}\right)$ which is regular in $S$, which is the desired sequence.

Define $k_{i}=\operatorname{deg}\left(f_{i}\right)$.

Proposition 6.7 The number of times the integer $m$ appears in the list $k_{1}, \ldots, k_{n}$ is precisely the number of roots in $\mathcal{R}^{+} \cap w^{-1}\left(\mathcal{R}^{-}\right)$of height $m-1$ minus the number of such roots of height $m$.

Proof Since the number of relations of degree $j$ from the original presentation of Akyıldız and Carrell which have been eliminated is the number of generators of degree $j$, the remaining number of relations is precisely the difference between these, which is also the number of roots of height $j / 2-1$ minus the number of height $j / 2$ in $\mathcal{R}^{+} \cap w^{-1}\left(\mathcal{R}^{-}\right)$.

Proof of Theorem 6.4 Applying the Hirsch lemma (as stated in the paper [5]) to the fibration $B \rightarrow G_{w} \rightarrow G_{w} / B$, we see that the cohomology ring $H^{*}\left(G_{w}\right)$ is the cohomology of the dg-algebra $H^{*}\left(G_{w} / B\right) \otimes_{S} \mathcal{K}_{T}$, where $\mathcal{K}_{T}$ is the Koszul complex of $S$ (the natural free resolution of $\mathbb{C}$ as an $S$-module). The complex $\mathcal{K}_{T}$ gains a dg-algebra structure from the Yoneda product. 
Since $\left(f_{1}, \ldots, f_{n}\right)$ is regular, $H^{*}\left(G_{w} / B\right)$ is quasi-isomorphic to the Koszul complex $\mathcal{K}_{\mathbf{f}}$. Thus, we have quasi-isomorphisms:

$$
H^{*}\left(G_{w} / B\right) \otimes_{S} \mathcal{K}_{T} \simeq \mathcal{K}_{\mathbf{f}} \otimes_{S} \mathcal{K}_{T} \simeq \mathcal{K}_{\mathbf{f}} \otimes_{S} \mathbb{C}
$$

The right-hand side is just an exterior algebra over $\mathbb{C}$ with generators $\gamma_{1}, \ldots, \gamma_{n}$ the degrees of which are given by $\operatorname{deg}\left(\gamma_{i}\right)=2 k_{i}-1$.

Since we used a Koszul complex, the generators $\gamma$ land in Hochschild degree 1 under the restriction map to $T$, so the degree of $\gamma_{i}$ is $\left(2 k_{i}, 1\right)$.

\section{References}

[1] E Akylldız, J B Carrell, A generalization of the Kostant-Macdonald identity, Proc. Nat. Acad. Sci. U.S.A. 86 (1989) 3934-3937 MR998938

[2] H H Andersen, C Stroppel, Twisting functors on $\mathcal{O}$, Represent. Theory 7 (2003) 681-699 MR2032059

[3] A A Bělinson, J Bernstein, P Deligne, Faisceaux pervers, from: "Analysis and topology on singular spaces, I (Luminy, 1981)", Astérisque 100, Soc. Math. France, Paris (1982) 5-171 MR751966

[4] J Bernstein, V Lunts, Equivariant sheaves and functors, Lecture Notes in Math. 1578, Springer, Berlin (1994) MR1299527

[5] P Deligne, P Griffiths, J Morgan, D Sullivan, Real homotopy theory of Kähler manifolds, Invent. Math. 29 (1975) 245-274 MR0382702

[6] M Goresky, R Kottwitz, R MacPherson, Equivariant cohomology, Koszul duality, and the localization theorem, Invent. Math. 131 (1998) 25-83 MR1489894

[7] M Goresky, R MacPherson, Intersection homology theory, Topology 19 (1980) 135162 MR572580

[8] V F R Jones, Hecke algebra representations of braid groups and link polynomials, Ann. of Math. (2) 126 (1987) 335-388 MR908150

[9] O Khomenko, V Mazorchuk, On Arkhipov's and Enright's functors, Math. Z. 249 (2005) 357-386 MR2115448

[10] M Khovanov, Matrix factorizations and link homology II arXiv:math.QA/0505056

[11] M Khovanov, Triply-graded link homology and Hochschild homology of Soergel bimodules, Internat. J. Math. 18 (2007) 869-885 MR2339573 arXiv:math.GT/0510265

[12] J Rasmussen, Some differentials on Khovanov-Rozansky homology arXiv: math.GT/0607544

[13] R Rouquier, Categorification of the braid groups arXiv:math.RT/0409593 
[14] W Soergel, Langlands' philosophy and Koszul duality, from: "Algebra-representation theory (Constanta, 2000)", NATO Sci. Ser. II Math. Phys. Chem. 28, Kluwer Acad. Publ., Dordrecht (2001) 379-414 MR1858045

[15] W Soergel, Kazhdan-Lusztig-Polynome und unzerlegbare Bimoduln über Polynomringen, J. Inst. Math. Jussieu 6 (2007) 501-525 MR2329762

[16] B Webster, Khovanov-Rozansky homology via a canopolis formalism, Algebr. Geom. Topol. 7 (2007) 673-699 MR2308960

Department of Mathematics, Massachusetts Institute of Technology

77 Massachusetts Avenue, Cambridge, MA 02139

Mathematisches Institut der Universität Freiburg

Freiburg 79106, Germany

bwebster@math.mit.edu, geordie.williamson@math.uni-freiburg.de

http://math.mit.edu/ bwebster, http://home.mathematik.uni-freiburg.de/ geordie/

Proposed: Vaughan Jones

Received: 8 August 2007

Seconded: Rob Kirby, Ralph Cohen

Revised: 19 December 2007

Geometry 83 Topology, Volume 12 (2008) 